\title{
Adenoid Cystic Carcinoma Invade the Tracheal Prominence: A Case Report and Literature Review
}

\author{
Kaiwen He, Haitao Huang, Shaomu Chen, Yu Feng, Haitao Ma* \\ Department of Thoracic Surgery, The First Affiliated Hospital of Soochow University, Suzhou, China \\ Email:*mht7403@163.com
}

How to cite this paper: He, K.W., Huang, H.T., Chen, S.M., Feng, Y. and Ma, H.T. (2021) Adenoid Cystic Carcinoma Invade the Tracheal Prominence: A Case Report and Literature Review. Case Reports in Clinical Medicine, 10, 8-16.

https://doi.org/10.4236/crcm.2021.101002

Received: December 11, 2020

Accepted: January 22, 2021

Published: January 25, 2021

Copyright $\odot 2021$ by author(s) and Scientific Research Publishing Inc. This work is licensed under the Creative Commons Attribution International License (CC BY 4.0).

http://creativecommons.org/licenses/by/4.0/

\begin{abstract}
Background: Adenoid cystic carcinoma (ACC) refers to a salivary gland neoplasm. Tracheal adenoid cystic carcinoma that invades the tracheal prominence is a relatively rare tumor among thoracic diseases. Objective: To explore the epidemiology, diagnosis, treatment and future development of tracheal adenoid cystic carcinoma (TACC). Methods: With asymptomatic invasion of the tracheal prominence, a case of tracheal adenoid cystic carcinoma with asymptomatic invasion of the tracheal prominence was reported. The clinical management process and surgical methods were introduced, and related literature was reviewed and summarized. Results: The patient was admitted due to tracheal augmentation mass for half a month. Chest CT at admission displayed the soft tissue density shadow behind the tracheal augmentation process. After the completion of other examinations, tracheal tumor resection and tracheal reconstruction were performed. Postoperative routine pathology exhibited that it was TACC and without inguinal lymph node metastasis. Conclusion: TACC is a low-grade tracheal tumor with low incidence, delayed clinical manifestations and lack of specificity as well. CT and bronchoscopy are helpful for the diagnosis of suspected patients. Meanwhile, radical surgical resection is the first choice of treatment. Adjuvant therapy can improve the therapeutic effect. Targeted immunotherapy is the developing direction of treatment.
\end{abstract}

\section{Keywords}

Tracheal Adenoid Cystic Carcinoma, Diagnosis, Treatment

\section{Introduction}

Primary tracheal tumors are much less common than bronchial and lung tu- 
mors. It has a wide variety, and about $90 \%$ of primary tracheal tumors are malignant in adults [1], of which primary adenoid cystic carcinoma and squamous cell carcinoma of the trachea are the most common histological types of tracheal malignancies, accounting for over $60 \%$. Tracheal adenoid cystic carcinoma (TACC) is a salivary gland and derived from bronchial mucosa or submucosal glands. Due to the late-onset and lack of specific clinical manifestations, it is often misdiagnosed or delayed by clinicians [2]. At present, surgical treatment is the choice for tracheal adenoid cystic carcinoma. This paper reports the diagnosis and management of patients with asymptomatic primary tracheal adenoid cystic carcinoma invading the augmentation process, and also elaborates the current treatment of TACC and the direction of future development.

\section{Clinical Data}

A 52-year-old male was examined by chest CT for nasal polypectomy, which revealed a mass-like soft tissue density shadow behind the tracheal prominence, measuring about $2.6 \times 2.4 \mathrm{~cm}$, narrowing of the initial segments of both bronchi, and with multiple slightly large lymph node shadows in the mediastinum (Figure 1). The patient had occasional chest distress symptoms and a 30-year history of smoking, one pack per day. Bronchoscopy proved invading the tracheal prominence, smooth surface, significant stenosis of the opening of the right common branch, and slight stenosis of the left common branch (Figure 2). Pathology revealed the adenoid cystic carcinoma of the tracheal carina. Three-dimensional reconstruction of the trachea was performed before surgery (Figure 3), which indicated that the size of the lesion was $31 \times 21 \times 20 \mathrm{~mm}$, the invasion of the right main trachea was severe, and the lesion protruded at the same time. The upper edge of the main trachea of the lesion was $20 \mathrm{~mm}$ away from the lower edge of the right main trachea. Bone scan displayed no obvious sign of bone metastasis. Pulmonary function was assessed by spirometry with FEV1 2.29 L, $69.1 \%$ predicted, and MVV 84\% predicted. Routine preoperative examination of the patient revealed no significant surgical contraindications and the primary tumour stage was T2. The patient was informed before being reported.

On May 13, 2020, the patient underwent tracheal tumor resection and reconstruction under general anesthesia smoothly. The patient experienced a right anterolateral fourth intercostal incision for chest entry, and a mass was observed at the near carina of the tracheal membrane, measuring about $3 \times 2 \times 2 \mathrm{~cm}$, that is consistent with preoperative three-dimensional reconstruction. The lower pulmonary ligament was released, the pericardium was opened, the lymph nodes were dissected, the left and right main bronchi were fully freed, the main trachea and left and right main trachea were cut $3 \mathrm{~mm}$ from the tumor with a blade (Figure 4), the spring endotracheal tube was intubated in the right main trachea, the right lung was ventilated, the specimen was removed, and rapid pathology revealed that the tracheal resection margin was not involved by cancer. The left main bronchus and main trachea were continuously sutured with a 4-0 pronelne suture and with the ends being aligned to tighten the anastomosis (Figure 5). 


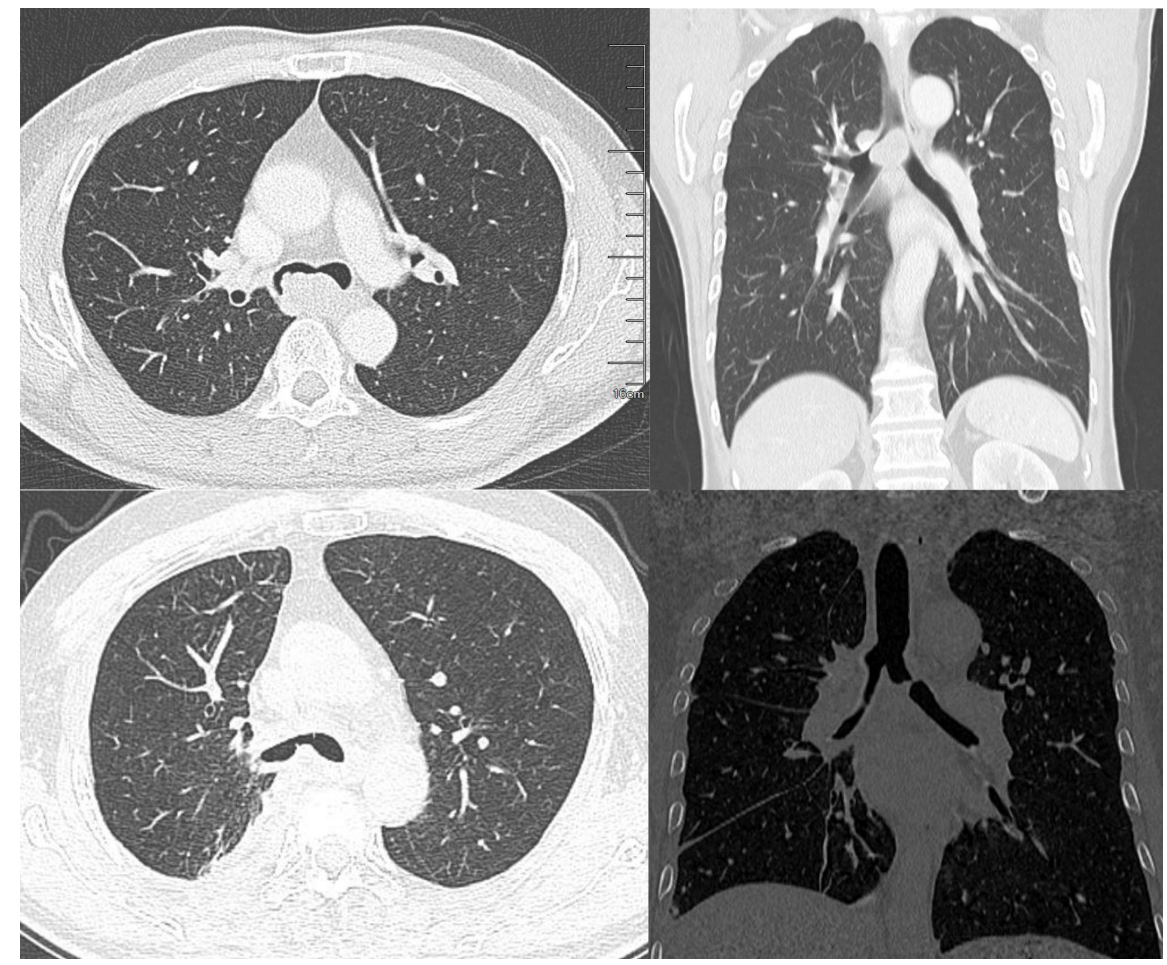

Figure 1. Preoperative CT image of chest and postoperative CT image of chest.

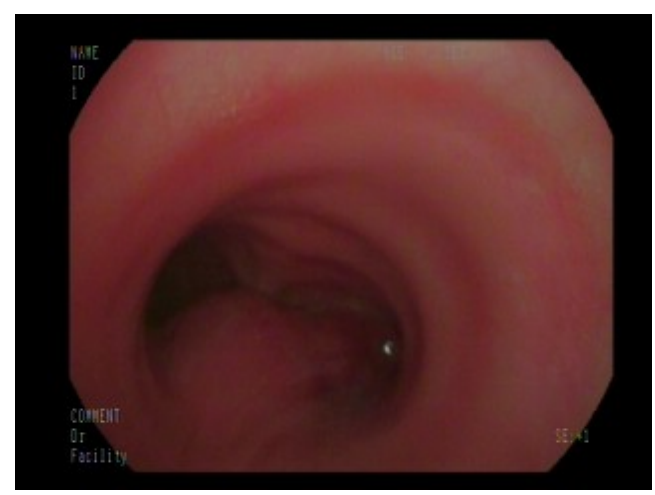

Figure 2. Bronchoscopy.
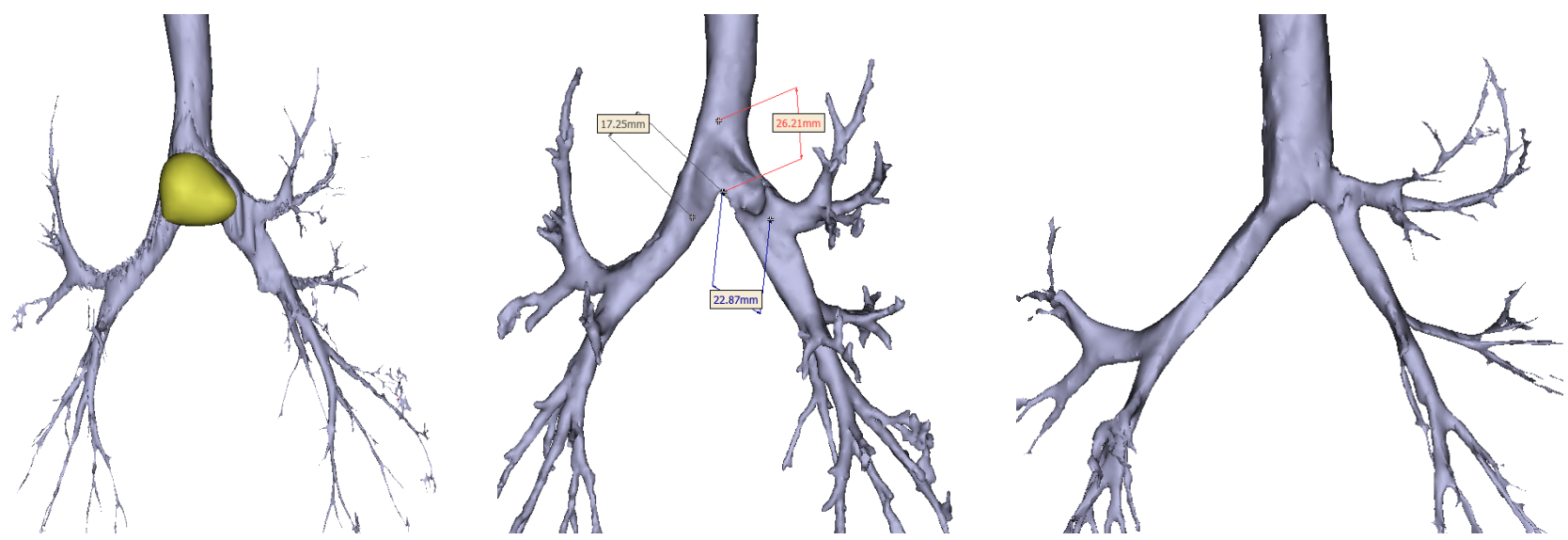

Figure 3. Preoperative and Postoperative three-dimensional reconstruction of trachea. 


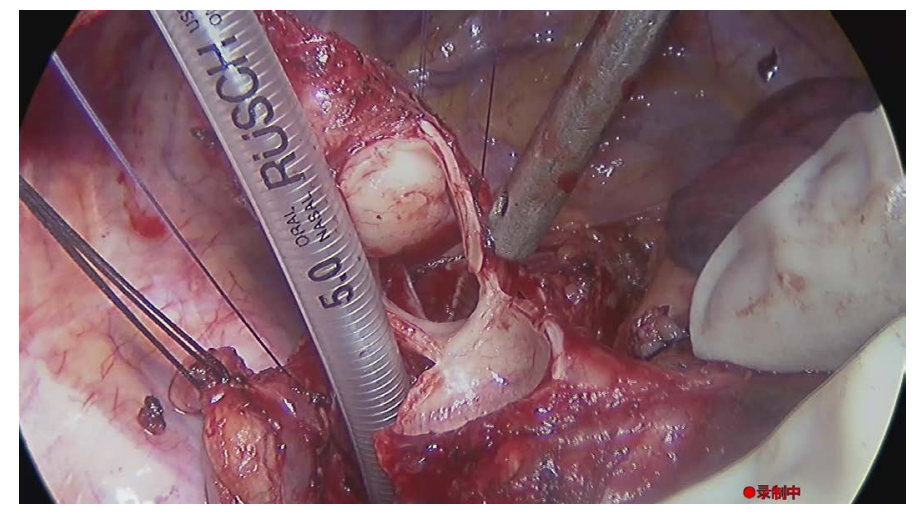

Figure 4. Intraoperative situation: Tumor and Right lung ventilation.

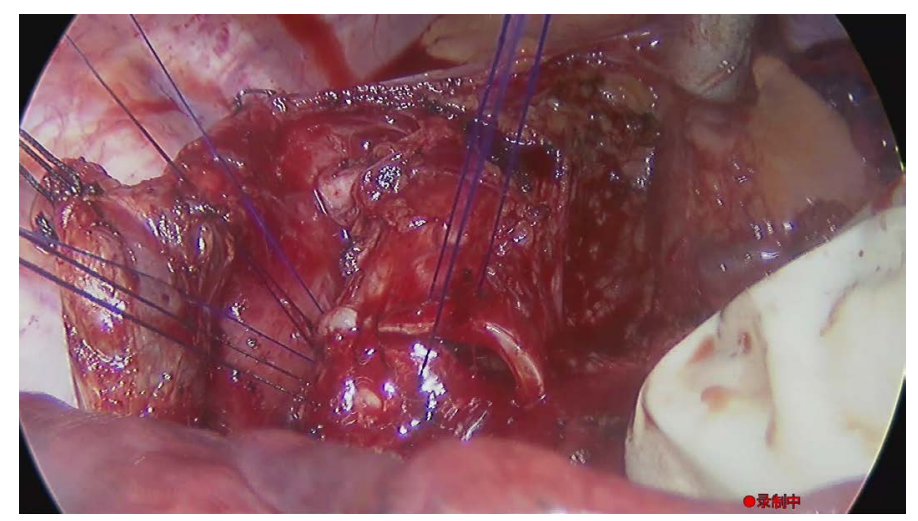

Figure 5. Intraoperative situation: Tracheal anastomosis.

The spring trachea was inserted into the sutured left tracheal lumen, and the left lung was changed for ventilation. The cartilage ring of the right wall of the trachea was fenestrated $1 \mathrm{~cm}$ above the anastomosis, so as to avoid damage to the tracheal membrane. The size of the fenestration was $1.5 \times 1 \mathrm{~cm}$. The right main bronchus and trachea were continuously sutured with 4-0 pronelne suture. The ends were aligned to tighten the anastomosis. The knot was tightened after confirming that there was no leakage at the anastomosis. The pedicled intercostal muscle was applied to wrap the anastomosis. One No. 28 and one No. 21 thoracic drainage tubes were placed in the seventh intercostal anterior axillary line and the midaxillary line, respectively. The chest layer was closed by layer after hemostasis and counting was correct. It was fixed with line 7 at the mandible and sternocleidomastoid muscle to flex the patient's neck, limit neck movement, and avoid excessive anastomotic orotracheal pressure. The operation went smoothly, with intraoperative about $200 \mathrm{ml}$ of blood loss.

\section{Surgical Results}

The patient recovered steadily after surgery, had no serious perioperative complications, and was discharged uneventfully. Postoperative routine pathology revealed adenoid cystic carcinoma (Figure 6) with negative tracheal resection margins and no metastasis was found in LN4, 5, 7, and 10. 


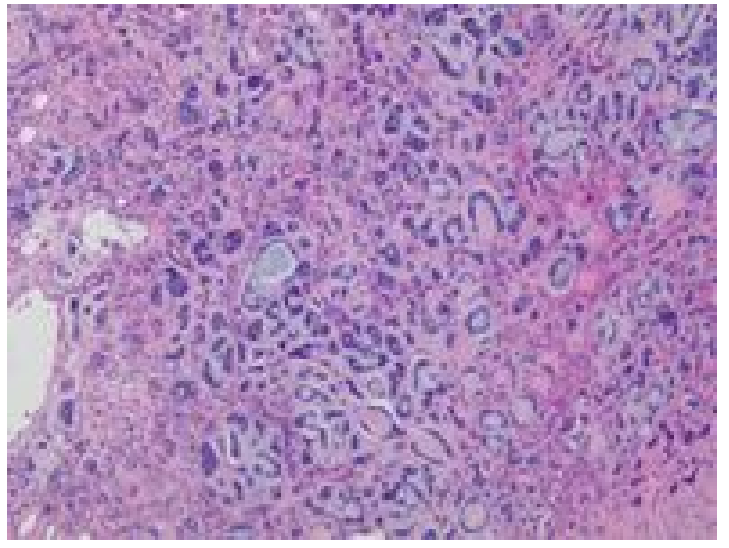

Figure 6. Pathological section of tumor $(\mathrm{HE} \times 40)$.

One month after surgery, the patient's general condition recovered well and no further subsequent treatment was provided. There was no recurrence after six months of follow-up.

The key factors of management include preoperative comprehensive evaluation, clear nature of the lesion by $\mathrm{CT}$ and bronchoscopic biopsy, display the structure and upper and lower boundaries of the lesion by preoperative bronchial three-dimensional reconstruction, fully understand the tumor growth, clarify the extent of surgical resection, and contribute to the accurate localization of the lesion before surgery. Three-dimensional reconstruction of the bronchus of this patient illustrated that: the size of the lesion was $31 \times 21 \times 20 \mathrm{~mm}$, the tumor involved more right main trachea, the upper pole of the tumor was convex to the main trachea, $26 \mathrm{~mm}$ away from the carina, the right and left main trachea incisal edges were 22 and $17 \mathrm{~mm}$ away from the carina (the mass was resected $3 \mathrm{~mm}$ away from the upper and lower boundary of the tumor to ensure the negative resection margin), the patient was fully freed during the operation, the tracheal wall of the right main trachea was fenestrated, and end-to-side anastomosis was performed, with good anastomosis, thus avoiding the right pneumonectomy, which was conducive to the preservation of pulmonary function. Postoperative chest CT was reexamined in patients, and three-dimensional reconstruction of the trachea demonstrated good surgical results (Figure 3).

\section{Discussion}

Primary bronchial adenoid cystic carcinoma is a rare salivary gland malignant tumor [3], and it originates from the bronchial mucosa or submucosal glands. About $50 \%$ of the disease occurs in the upper third of the trachea.

Adenoid cystic carcinoma of the trachea lacks specific clinical manifestations, including irritable cough and expectoration in the early stage and persistent symptoms. Bronchodilator and glucocorticoid treatment also has certain effects and is often misdiagnosed as pulmonary infection, chronic bronchitis, chronic obstructive pulmonary disease, bronchial asthma, etc. Delayed asthma and dyspnea occur only after $50 \%$ of the tracheal lumen are blocked in the middle 
and late stages [4]. Delayed clinical manifestations are often misdiagnosed as chronic obstructive pulmonary disease, bronchial asthma, or delayed diagnosis by clinicians [5]. Tracheal adenoid cystic carcinoma presents in middle-aged patients with an average distribution between men and women, and smoking is not a risk factor for it [6]. Among the auxiliary images, CT and three-dimensional reconstruction play certain advantages in the evaluation of airway tumors [7], PET-CT is also helpful to determine the stage of the disease [8] and is adopted to guide adjuvant radiotherapy, and bronchoscopic biopsy can provide a definite histological diagnosis as well.

Radical surgical resection is preferred for patients in good general condition and can tolerate surgery and meet safe tension-free endotracheal anastomosis [9], so as to provide complete resection of pathology and restoration of airway continuity, while patients have high postoperative morbidity and mortality [10], which is technically challenging for most medical centers. Tracheal reconstruction methods that invade the carina, thus resulting in stenosis and preserving the lung parenchyma are end-to-end anastomosis and end-to-side anastomosis, and the choice of surgical approach is determined based on the location and size of the tumor (Figure 7).

Surgical treatment of airway tumors requires resection as much as possible. However, owing to anatomical limitations, many patients cannot provide complete resection of the pathology during surgery. Preoperative three-dimensional reconstruction has greater advantages than two-dimensional image examination, because it can simulate and display the surgical anatomy, determine the surgical margin and ensure the negative margin. Consequently, routine preoperative three-dimensional reconstruction may have a better role in terms of evaluating and guiding airway tumors, and helping to improve the success rate of surgical resection of airway tumors with negative margins and increase the benefit rate of patients.

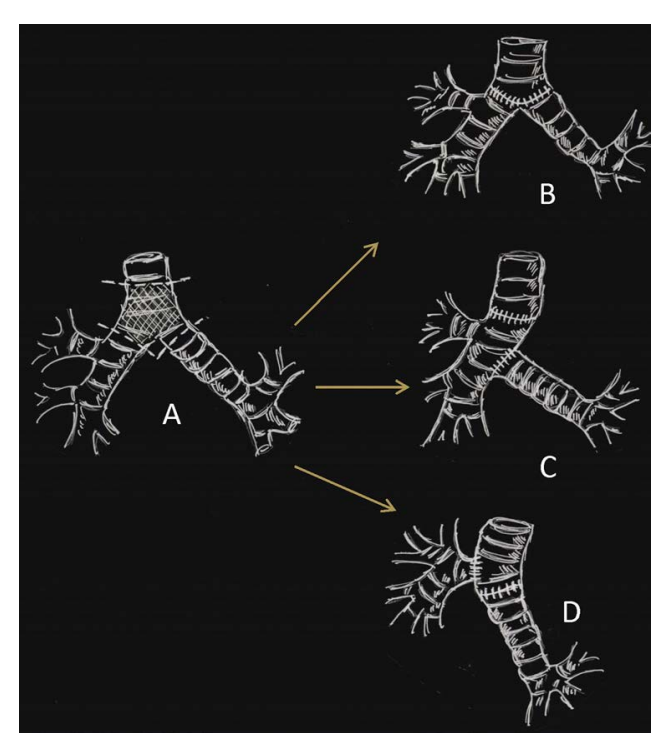

Figure 7. Tracheal reconstruction demonstration. 
As for patients limited by tracheal anatomy (usually tumors are larger than 4 $\mathrm{cm}$ [11], or, in some cases tracheal resection length is more than $6 \mathrm{~cm}$ and cannot ensure tracheal blood circulation and anastomotic tension [5]), tumor invasion of the surrounding trachea or tissues, intolerance to surgery (advanced age, severe complications), the treatment goal is airway recanalization, adjuvant therapy can control tumor progression, and tumor reduction can be achieved by bronchoscopic techniques, including electrocoagulation, thermal ablation therapy, and tracheal stent placement. In this case, preoperative three-dimensional reconstruction of the bronchus successfully showed the extent of lesion involvement, with $26 \mathrm{~mm}$ from the carina. The resection margins of the right and left main trachea were 22 and $17 \mathrm{~mm}$ from the carina into the main tracheal lumen, respectively. Meanwhile, the resection of the mass $3 \mathrm{~mm}$ from the upper and lower boundaries of the tumor during surgery could ensure negative resection margins and meet the surgical requirements.

TACC has an occult invasion of surrounding collagen fibers or neurovascular bundles, and invasion to the surrounding, with many local recurrences after surgery. Maziak DE et al. proposed that postoperative and combined radiotherapy can appropriately control the disease [12] and was proved to have a statistically significant survival rate in patients with primary ACC who cannot experience surgery and receive radiotherapy or palliative radiotherapy, with a five-year follow-up survival rate of $91 \%$, which can be applied as postoperative enhanced therapy or adjuvant therapy for local recurrence. Haitang Yang et al. put forward that radiation therapy should be adopted for adenoid cancer, even in patients with negative margins [13]. Endotracheal brachytherapy is reported to be applied to enhance the effects of primary tumor or palliative late treatment [14]. Combined with adjuvant multislice spiral CT, it can enhance the effects of radiotherapy for the lesion, while avoiding the harm that is caused by irradiation to normal tissues. However, there is a lack of multicenter prospective study support for the selection of radiotherapy dose, and there is no clear radiotherapy index. Grillo recommends radiotherapy at a dose of 4500 - 6500 cGy after the resection of adenoid cystic carcinoma. However, Furong Chen et al. reported that adjuvant radiotherapy after the incomplete resection did not additionally increase patient DFS and OS values [15]. Data on the availability of chemotherapy for TACC is scarce, and there is no evidence that chemotherapy can be adopted to treat tracheal ACC. Manninen M et al. indicated that 28 patients who received cytotoxic therapy did not respond to chemotherapy [16]. In a retrospective study, the addition of chemotherapy to postoperative radiotherapy had no benefit on overall survival.

In the past, tracheal tumors were considered unresectable. In 1946, Besley successfully completed augmentation and distal tracheotomy for the first time. With the continuous development of surgical techniques, fast-track thoracoscopic resection of tracheal tumors was advocated to be achieved. At the same time, relevant studies on tracheal allograft transplantation and autologous tra- 
cheal replacement are also ongoing [17]. Targeted therapy continues to evolve, and cell lines that show the characteristics of adenoid cystic carcinoma stem cells are cultured. NOTCH1, SOX10, and FABP7 drive the induction of adenoid cystic carcinoma stem cell death, thus providing the possibility for targeted therapy of bronchial adenoid cystic carcinoma [18].

\section{Data Availability}

The data used to support this study are restricted to protect patient privacy.

\section{Patient Consent for Publication}

Written informed consent for publication of the present report was obtained from the patient.

\section{Authors' Contributions}

Haitao Ma conceived and designed this case report. Kaiwen $\mathrm{He}$ is the first author, wrote the initial draft of the report. All authors have read and approved the final version of the manuscript.

\section{Ethics Approval and Consent to Participate}

Written informed consent for surgery was obtained from the patient.

\section{Conflicts of Interest}

The authors declare that there are no conflicts of interest regarding the publication of this article.

\section{References}

[1] Madariaga, M.L. and Gaissert, H.A. (2018) Secondary Tracheal Tumors: A Systematic Review. Annals of Cardiothoracic Surgery, 7, 183-196. https://doi.org/10.21037/acs.2018.02.01

[2] Yildirim, E. (2018) Principles of Urgent Management of Acute Airway Obstruction. Thoracic Surgery Clinics, 28, 415-428. https://doi.org/10.1016/j.thorsurg.2018.05.006

[3] Moran, C.A., Suster, S. and Koss, M.N. (1994) Primary Adenoid Cystic Carcinoma of the Lung. A Clinicopathologic and Immunohistochemical Study of 16 Cases. Cancer, 73, 1390-1397.

[4] Sherani, K., Vakil, A., Dodhia, C. and Fein, A. (2015) Malignant Tracheal Tumors: A Review of Current Diagnostic and Management Strategies. Current Opinion in Pulmonary Medicine, 21, 322-326. https://doi.org/10.1097/MCP.0000000000000181

[5] Lancaster, T.S., Krantz, S.B. and Patterson, G.A. (2016) Tracheal Resection with Carinal Reconstruction for Squamous Cell Carcinoma. The Annals of Thoracic Surgery, 102, e77-e79. https://doi.org/10.1016/j.athoracsur.2016.02.070

[6] Grillo, H.C. and Mathisen, D.J. (1990) Primary Tracheal Tumors: Treatment and Results. The Annals of Thoracic Surgery, 49, 69-77. https://doi.org/10.1016/0003-4975(90)90358-D

[7] McInnis, M.C., Weisbrod, G. and Schmidt, H. (2018) Advanced Technologies for 
Imaging and Visualization of the Tracheobronchial Tree: From Computed Tomography and MRI to Virtual Endoscopy. Thoracic Surgery Clinics, 28, 127-137. https://doi.org/10.1016/j.thorsurg.2018.01.005

[8] Shepard, J.O., Flores, E.J. and Abbott, G.F. (2018) Imaging of the Trachea. Annals of Cardiothoracic Surgery, 7, 197-209. https://doi.org/10.21037/acs.2018.03.09

[9] Belsey, R. (1946) Stainless Steel Wire Suture Technique in Thoracic Surgery. Thorax, 1, 39-47. https://doi.org/10.1136/thx.1.1.39

[10] Mitchell, J.D., Mathisen, D.J., Wright, C.D., et al. (1999) Clinical Experience with Carinal Resection. Journal of Thoracic and Cardiovascular Surgery, 117, 39-52; discussion 52-53. https://doi.org/10.1016/S0022-5223(99)70468-X

[11] Blasberg, J.D. and Wright, C.D. (2012) Surgical Considerations in Tracheal and Carinal Resection. Seminars in Cardiothoracic and Vascular Anesthesia, 16, 190-195. https://doi.org/10.1177/1089253212450342

[12] Maziak, D.E., Todd, T.R.J., Keshavjee, S.H., Winton, T.L., Van Nostrand, P. and Pearson, F.G. (1996) Adenoid Cystic Carcinoma of the Airway: Thirty-Two Year Experience. Journal of Thoracic and Cardiovascular Surgery, 112, 1522-1532. https://doi.org/10.1016/S0022-5223(96)70011-9

[13] Kaminski, J.M., et al. (2003) The Role of Radiation Therapy and Chemotherapy in the Management of Airway Tumors Other Than Small-Cell Carcinoma and Non-Small-Cell Carcinoma. Chest Surgery Clinics of North America, 13, 149-167. https://doi.org/10.1016/S1052-3359(02)00040-6

[14] Alongi, F., Di Muzio, N., Motta, M., Broggi, S., De Martin, E., Bolognesi, A., et al. (2008) Adenoid Cystic Carcinoma of Trachea Treated with Adjuvant Hypofractionated Tomotherapy. Case Report and Literature Review. Tumori Journal, 94, 121-125. https://doi.org/10.1177/030089160809400122

[15] Chen, F., Huang, M., Xu, Y., Li, T., Xie, K., Zhang, L., et al. (2015) International Journal of Clinical Oncology, 20, 686-692.

https://doi.org/10.1007/s10147-014-0771-6

[16] Manninen, M., Pukander, J.S., Flander, M.K., Laippala, P.J., Huhtala, H.S.A. and Karma, P.H. (1993) Treatment of Primary Tracheal Carcinoma in Finland in 1967-1985. Acta Oncologica, 32, 277-282. https://doi.org/10.3109/02841869309093595

[17] Martinod, E., Chouahnia, K., Chouahnia, D.M., et al. (2018) Feasibility of Bioengineered Tracheal and Bronchial Reconstruction Using Stented Aortic Matrices. JAMA, 319, 2212-2222. https://doi.org/10.1001/jama.2018.4653

[18] Panaccione, A., Chang, M.T., Carbone, B.E., Guo, Y., Moskaluk, C.A., Virk, R.K., et al. (2016) NOTCH1 and SOX10 Are Essential for Proliferation and Radiation Resistance of Cancer Stem-Like Cells in Adenoid Cystic Carcinoma. Clinical Cancer Research, 22, 2083-2095. https://doi.org/10.1158/1078-0432.CCR-15-2208 